\title{
Habilidades funcionais de crianças com deficiências em inclusão escolar - barreiras para uma inclusão efetiva
}

\author{
Functional skills of children with deficiencies \\ in school inclusion - barriers to effective inclusion
}

Fernanda Moreira Teles ${ }^{1}$

Rosa Resegue $^{1}$

Rosana Fiorini Puccini ${ }^{1}$
${ }^{1}$ Departamento de

Pediatria, Escola Paulista de

Medicina. Unifesp. R. Sena

Madureira 1500, Vila

Mariana. 04.021-001 São

Paulo SP.

fernandamteles@ig.com.br

\begin{abstract}
The scope of this article is to describe the disabilities of students in the process of inclusion by means of the PEDI - Pediatric Evaluation of Disability Inventory. A cross-sectional analysis was conducted. The population studied included 181 students (7-10 years old) from 31 public schools in São Paulo. Using a questionnaire applied to the caregiver, 197 activities were evaluated in the areas of self-care, mobility and social function. In accordance with the PEDI, differences $(p<0.05)$ in student performance were observed between grades in the scales of self-care (average of $77.8 \%$ in the 1 st grade and $95.2 \%$ in the 4th grade) and mobility (average of $90 \%$ in the 1 st grade and $99.8 \%$ in the 4 th grade); social function had the lowest averages, except for those with physical disabilities, in which the most affected area was mobility (average of 62.7\%). The PEDI enabled the identification of disabilities per group and individual disability, which may constitute an important tool for targeted intervention and follow-up at school.
\end{abstract}

Key words Health of people with deficiencies or disabilities, Children with deficiencies, Rehabilitation, Daily activities
Resumo O objetivo deste artigo é descrever as incapacidades de alunos em processo de inclusão por meio do PEDI (Inventário de Avaliação Pediátrica de Incapacidades). Estudo transversal analítico. População: 181 alunos (7-10 anos) de 31 escolas municipais de São Paulo. Por meio de questionário aplicado ao cuidador, foram avaliadas 197 atividades nas áreas de autocuidado, mobilidade e função social. Diferenças $(p<0,05)$ no desempenho dos alunos, segundo o PEDI, foram observadas entre as séries nas escalas de autocuidado (média de 77,8\% na $1^{a}$ série e de $95,2 \%$ na $4^{a}$ série) e mobilidade (média de 90,0\% na $1^{a}$ série e 99,8\% na $4^{a}$ série); a função social apresentou as menores médias, exceção ao grupo com deficiência física, cuja área mais afetada foi a mobilidade (média de 62,7\%). O PEDI permitiu a identificação de incapacidades por grupo de deficiências e individual, podendo constituir instrumento importante para intervenção direcionada e acompanhamento na escola.

Palavras-chave Saúde da pessoa com deficiência ou incapacidade, Crianças com deficiência, Reabilitação, Atividades cotidianas 


\section{Introdução}

Inclusão é o movimento da sociedade que visa a produzir a igualdade de oportunidades para todos. Quando focada sob a ótica individual, a inclusão supõe que cada um tenha a oportunidade de fazer suas próprias escolhas e construir sua identidade pessoal e social ${ }^{1}$. A prática da inclusão social vem aos poucos substituindo a da integração social e parte do princípio de que para inserir todas as pessoas a sociedade deve ser modificada de modo $a$ atender às necessidades de seus membros - uma sociedade inclusiva não admite preconceitos, discriminações, barreiras sociais, culturais e pessoais. Neste sentido, a inclusão social da pessoa com deficiência significa possibilitar a ela, respeitando as necessidades próprias da sua condição, o acesso aos serviços públicos, aos bens culturais e aos produtos decorrentes do avanço social, político, econômico e tecnológico da sociedade ${ }^{2}$.

Com base nesse conceito mais global, a educação inclusiva é definida como conjunto de processos educacionais decorrentes da execução de políticas articuladas que impeçam qualquer forma de segregação ou isolamento. Essas políticas buscam favorecer o acesso à escola regular, ampliar a participação e assegurar a permanência de todos os alunos, independentemente de suas particularidades. Sob o ponto de vista prático, a educação inclusiva garante a qualquer criança o acesso ao ensino fundamental, nível de escolaridade obrigatório a todo cidadão brasileiro ${ }^{1}$, tomando como referência movimentos que ocorrem mundialmente nas últimas décadas.

De acordo com a Declaração de Salamanca, "as necessidades educativas especiais incorporam os princípios já provados de uma pedagogia equilibrada que beneficia todas as crianças". Parte do princípio de que a diversidade é uma característica humana e, nesta perspectiva, a aprendizagem deve ajustar-se às necessidades individuais, substituindo a concepção de que cada criança deve se adaptar aos supostos princípios quanto ao ritmo e à natureza do processo educativo. Uma pedagogia centrada na criança é positiva para todos os alunos e, consequentemente, para toda a sociedade. As escolas com propostas pedagógicas centradas na criança constituem a base para a construção de uma sociedade que valoriza as pessoas, que respeita a dignidade e as diferenças dos seres humanos ${ }^{3}$.

No Brasil, a Lei de Diretrizes e Bases da Educação Nacional (lei n. 9.394/96) para a educação especial na educação básica estabelece a seguinte definição para educando com necessidades edu- cacionais especiais: "educando com dificuldades acentuadas de aprendizagem ou limitações no processo de desenvolvimento que dificultam o acompanhamento das atividades curriculares vinculadas a uma causa orgânica específica ou relacionadas a condições, disfunções, limitações ou deficiências; ou, dificuldades de comunicação e sinalização diferenciadas dos demais alunos, demandando a utilização de linguagens e códigos aplicáveis; ou, altas habilidades/superdotação, grande facilidade de aprendizagem que o leve a dominar rapidamente conceitos, procedimentos e atitudes".

De acordo com o censo demográfico do IBGE (2000), 14,5\% da população brasileira referiu algum tipo de deficiência. Este número, bastante superior aos dados dos censos anteriores, decorreu em grande parte da utilização de um conceito mais amplo de deficiência, o qual leva em consideração, além da incapacidade, a presença e a intensidade de limitações funcionais e da inclusão de faixas etárias. Levantamento realizado pela área temática "Saúde da Pessoa com Deficiência", da Secretaria Municipal da Saúde de São Paulo, constatou que o município, com uma população de cerca de 11 milhões de habitantes, apresenta $10,3 \%$ de pessoas com deficiência, sendo $1,3 \%$ do tipo mental, $2,4 \%$ auditiva, $3,3 \%$ motora, $0,7 \%$ física e $6,6 \%$ visual $^{5}$.

Qualquer julgamento da deficiência está sujeito a vieses de diferentes naturezas, sendo necessária a realização de avaliações multidisciplinares, que possam oferecer informações que contribuam para minimizar os aspectos excludentes. Qualquer que seja a opção assumida para a construção de uma sociedade inclusiva, a gravidade do acometimento envolve questões que precisam ser analisadas profundamente. O que torna a pessoa deficiente aos olhos sociais não é apenas a deficiência ou déficit que ele apresenta, mas o significado dessa condição desviante. $\mathrm{O}$ resultado final da deficiência decorre de restrições de participação relacionadas à interação de inúmeros fatores ${ }^{6-8}$.

Definida a política de inclusão, os sistemas de educação foram solicitados a se organizar na sociedade, considerando todas suas implicações para as escolas, professores, alunos e pais ${ }^{9}$. Nesse processo, há a necessidade de compreender o verdadeiro papel da escola, reconhecendo seus limites. Se por um lado, ela não pode assumir funções específicas de outras áreas, como a saúde e a assistência social, por outro, não pode se eximir de sua verdadeira função, a pedagógica ${ }^{5}$. Para contribuir nesse processo, é fundamental 
que a escola identifique e quantifique as dificuldades de uma criança visando à viabilização de um programa mais adequado, favorecendo o processo de sua inclusão no grupo. Pensar a educação na atualidade exige, essencialmente, refletir sobre a sociedade da qual ela faz parte e os princípios que regem essa sociedade e seus processos educativos ${ }^{10}$.

Considerando a dificuldade do educador em conhecer efetivamente as necessidades de todos os seus alunos, a junção de conhecimentos e habilidades de profissionais especializados em estratégias instrucionais, abordagens de aprendizagem cooperativa, problemas comportamentais e práticas de avaliação, entre outros saberes, poderiam propiciar a criação coletiva de escolas mais efetivas ${ }^{11}$.

Diante das dificuldades das crianças com deficiência integrarem-se ao grupo escolar, considera-se importante que, por meio de um instrumento de avaliação já validado, possam ser oferecidas informações e subsídios que contribuam para que a escola e a família dessas crianças processem a inclusão. Compreende-se que este processo deva ser compartilhado, buscando reduzir ao máximo as dificuldades que elas deverão enfrentar devido às barreiras arquitetônicas, necessidades de ajuda para realização de atividades da vida diária e outros aspectos sociais.

Por estas razões, o presente estudo buscou, por meio do Inventário de Avaliação Pediátrica de Incapacidade (PEDI), identificar as incapacidades que possam dificultar o processo de inclusão escolar e fornecer informações que possam colaborar com o planejamento escolar, visando à superação dessas dificuldades. Outros trabalhos que utilizaram o PEDI puderam fornecer informações importantes sobre o desempenho de atividades funcionais e, portanto, apontaram para as possibilidades de intervenções mais direcionadas à área de maior comprometimento ${ }^{12}$. Sua utilização tem ocorrido em diferentes grupos e seus resultados têm trazido informações mais precisas, quantificando necessidades e direcionando as ações. Assim, este estudo teve como objetivo descrever as incapacidades de alunos em processo de inclusão em uma regional do município de São Paulo por meio do PEDI (Inventário de Avaliação Pediátrica de Incapacidade).

\section{Métodos}

Estudo transversal analítico realizado nas escolas de ensino fundamental da Prefeitura Municipal de São Paulo na Delegacia Regional de Ensino
(DRE) Distrito Penha, zona leste de São Paulo. A Secretaria Municipal de Educação do município de São Paulo está subdividida em 13 diretorias regionais de ensino. Estas são responsáveis pelo grupo de escolas de cada região e em cada uma delas há um serviço de apoio à inclusão - Centro Escola Família de Apoio à Inclusão (CEFAI), que tem como atribuições o acompanhamento dos alunos com deficiência por meio de visitas sistemáticas às escolas, avaliação pedagógica, reuniões com professores e coordenadores pedagógicos, atendimentos a pais e mapeamento dos atendimentos da região.

As escolas com maior número de crianças com necessidades especiais apresentam também salas de apoio à inclusão, denominadas Salas de Apoio e Acompanhamento à Inclusão (SAAI). Estas são destinadas ao atendimento educacional em caráter complementar, suplementar ou exclusivo aos alunos que apresentam algum tipo de deficiência.

De 33 escolas da DRE Distrito Penha, zona leste de São Paulo, 31 tinham crianças com deficiência matriculadas, as quais foram incluídas neste estudo. Os diretores ou orientadores pedagógicos destas escolas foram contatados pela pesquisadora e, antes de iniciar a aplicação do PEDI, foi realizada uma apresentação da pesquisa, seus objetivos e procedimentos. Nessa ocasião, a pesquisadora obteve informações sobre o processo de inclusão desenvolvido em cada escola - acessibilidade, qualificação dos professores, sistemática de reuniões com os pais, suas dificuldades, incluindo a discussão e a definição compartilhada sobre a progressão nas séries. A população avaliada foi constituída por crianças com idades de 7 a 10 anos em processo de inclusão escolar no ciclo Fundamental I - $1^{\text {a }}$ a $4^{\text {a }}$ série (antes da ampliação para nove anos), no ano de 2007. Foi considerado o total de crianças com deficiência que apresentava laudo diagnóstico; a lista de crianças foi fornecida pelo Centro de Educação e Formação de Apoio à Inclusão (CEFAI) da DER- Delegacia Regional Penha, totalizando 200 crianças em 2007. A partir da lista de alunos, os pais ou cuidadores foram convidados a participar da pesquisa; uma vez aceita a entrevista era agendada e realizada na escola pela pesquisadora. Do total de 200 crianças, foram excluídas 19: 13 cujos cuidadores não compareceram aos dois agendamentos para realização da entrevista, sem justificativas; 2 cuidadores não apresentaram condições para continuidade da pesquisa, pois não conseguiram responder com coerência às perguntas; 1 caso cujo cuidador é usuário de dro- 
gas de forma contínua (sem condição de responder ao questionário) e 3 cuidadores que se recusaram a participar. Assim, a população final do estudo foi constituída por 181 crianças.

Variáveis do estudo - idade (em anos), sexo, série e tipo de deficiência. Neste estudo, o tipo de deficiência considerou a nomenclatura adotada pela Secretaria Municipal de Educação e pelo CEFAI, a qual é descrita a seguir:

Conduta típica: crianças com diagnóstico médico de hiperatividade, doenças psiquiátricas e transtornos de comportamento e alterações das aquisições cognitivas, incluindo deficiência mental de todos os níveis.

DGD - deficiência global do desenvolvimento: crianças com autismo de todos os tipos (leve, moderado, grave) e Asperger. Este termo, ao invés de Transtorno Global do Desenvolvimento, é utilizado pela SME e corresponde ao código F84 - CID 10.

Deficiência física: deficiências por falta de membros, assimetria de membros, assim como deformidades ósseas e deficiências motoras.

Síndromes Genéticas: grupo com laudo de síndromes genéticas.

Síndrome de Down (SD): por se tratar de um grupo maior e com características específicas a Síndrome de Down foi separada das demais.

Múltiplas deficiências: crianças com dois ou mais comprometimentos. Neste grupo, são colocadas as crianças que apresentam deficiências associadas a qualquer outra alteração/doença. Exemplo: deficiência motora e epilepsia; deficiência auditiva e visual, entre outros.

Outras deficiências: doenças como Síndrome da Amplificação Dolorosa, deficiência visual, dislexia, distúrbios de fala e outros.

Por meio de questionário estruturado foi entrevistado o cuidador. Considerou-se como cuidador qualquer pessoa, independente do grau de parentesco, que acompanha a criança no seu cotidiano durante as atividades diárias. A avaliação de habilidades funcionais foi realizada utilizando-se o PEDI, validado para a população brasileira em 2005. A idade em que mais de $90 \%$ das crianças conseguiram executar determinada atividade encontra-se determinada e validada. Para sua aplicação, a pesquisadora, única a aplicar o instrumento, realizou o treinamento mínimo recomendado pelos autores. Embora possa ser aplicado sem a presença do paciente, neste estudo empregou-se o método de entrevista simultânea à observação direta da criança ${ }^{12}$.

Para a averiguação das habilidades funcionais, foram avaliadas 73 atividades de autocui- dado, 59 de mobilidade e 65 de função social, perfazendo um total de 197 para as quais a criança foi considerada capaz (1) ou incapaz (0). Valor 0 indica que a criança é "totalmente incapaz", ou seja, a criança não realiza a atividade e o valor 1 indica que a criança é "totalmente capaz", ou seja, ela realiza a atividade do subconjunto. São exemplos de atividades de autocuidado: utilização de utensílios para alimentação, utilização de recipientes para beber, higiene oral, lavar as mãos, controle urinário, controle intestinal. São exemplos de atividades de mobilidade: transferências no banheiro, transferências de cadeiras, transferências no carro, locomoção em ambiente interno, locomoção em ambiente externo (velocidade, distância). São exemplos de atividades da função social: compreensão do significado das palavras, compreensão de frases complexas, interação com companheiros, jogo social interativo, tarefas domésticas, autoproteção.

\section{Análise estatística}

Neste trabalho, utilizaram-se análises descritivas totais para cada sub-escala. Para as comparações ao longo do estudo, considerou-se a escala transformada - as originais transformadas em de 0 a 100 - possibilitando comparações com diferentes grandezas e, desta forma, facilitando a interpretação dos resultados, como sugerem os autores McDowell e Newell ${ }^{13}$.

$\underset{\text { escala }}{\text { transformada }}=\frac{\begin{array}{c}\text { (pontuação original }- \text { menor } \\ \text { pontuação possível) }\end{array}}{\text { amplitude da escala original }}$

Em todos os casos considerou-se o escore contínuo que fornece informações sobre o nível de capacidade da criança, não se levando em consideração a faixa etária da mesma.

Para as comparações entre algumas variáveis do estudo que possuíam duas categorias, o teste utilizado foi o t-Student, considerando um nível de significância de 5\%. Para a comparação entre os grupos das variáveis citadas, o teste utilizado foi a Análise de Variância (ANOVA). Para a utilização deste teste foi verificado se, para cada variável, as variâncias eram homogêneas entre os grupos. Quando não foi verificada sua homogeneidade, foi feito o ajuste através do teste de Brown-Forsythe (BF). Foi realizada comparação da pontuação obtida segundo os grupos de deficiência (diagnósticos agrupados) entre as diferentes áreas: autocuidado, mobilidade e função social. Havendo diferença entre as medidas foram 
realizadas comparações múltiplas (comparação dos diagnósticos dois a dois) para identificar quais categorias apresentaram diferenças entre si. Os testes utilizados para as comparações múltiplas foram: teste de Bonferroni ou o teste de Dunnett, este último quando foi necessário o ajuste de Brown-Forsythe (BF). Para estas comparações também foi considerado um nível de significância de 5\%.

Este estudo foi aprovado pelo CEFAI e pelo Comitê de Ética e Pesquisa da Universidade Federal de São Paulo. Os cuidadores assinaram o Termo de Consentimento Livre e Esclarecido.

\section{Resultados}

A distribuição por idade dos alunos foi: 7 anos 39 alunos, 8 anos -32 alunos, 9 anos -53 alunos

Tabela 1. Frequência dos diagnósticos dos alunos com deficiência, classificados e agrupados segundo a orientação da SME/CEFAI. DRE-Penha/São Paulo, 2007- 2009.

\begin{tabular}{lcr}
\hline \multicolumn{1}{c}{ Tipo de deficiência } & No de alunos & \multicolumn{1}{c}{$\%$} \\
\hline Conduta típica & 55 & 30,4 \\
Deficiência física & 27 & 14,9 \\
Deficiência Global do & 12 & 6,6 \\
Desenvolvimento & & \\
Múltiplas deficiências & 33 & 18,2 \\
Síndrome Down & 17 & 9,4 \\
Síndromes genéticas & 12 & 6,6 \\
Outras deficiências & 25 & 13,9 \\
Total & $\mathbf{1 8 1}$ & $\mathbf{1 0 0 , 0}$ \\
\hline
\end{tabular}

e maior que 10 anos - 53 alunos. Quanto à distribuição por séries: $1^{\text {a }}$ série -35 alunos, $2^{\text {a }}$ série 46 alunos, $3^{\text {a }}$ série -50 alunos e $4^{\text {a }}$ série -50 alunos.

Na Tabela 1, encontra-se a distribuição dos alunos classificados quanto ao tipo de deficiência, destacando-se a maior frequência para o grupo de conduta típica $(30,4 \%)$. Na Tabela 2, observa-se o desempenho obtido pelos alunos nas três áreas na escala original e transformada (padronizadas em pontuações de 0 a 100).

$\mathrm{Na}$ Tabela 3, encontram-se as análises de associação de desempenho nas áreas de autocuidado, mobilidade e função social, segundo a idade, verificando-se que não houve diferenças estatisticamente significantes. A Tabela 4 refere-se à série escolar, verificando-se progressivo desempenho nas áreas de autocuidado e mobilidade; na função social a melhora do desempenho não é tão acentuada e não há diferença estatisticamente significante.

Tabela 2. Desempenho dos alunos nas áreas de auto-cuidado, mobilidade e função social, segundo o PEDI - Inventário de Avaliação Pediátrica de Incapacidade. DRE-Penha/São Paulo, 2007-2009.

\begin{tabular}{lcc}
\hline \multicolumn{1}{c}{ Escala Original (Criança) } & Média & Dp \\
\hline Pontuação: Autocuidado & 64,0 & 13,4 \\
Pontuação: Mobilidade & 59,8 & 12,8 \\
Pontuação: Função Social & 47,1 & 12,5 \\
Escala transformada (Criança) & & \\
Pontuação: Autocuidado (0-100) & 87,7 & 18,4 \\
Pontuação: Mobilidade (0-100) & 93,4 & 20,0 \\
Pontuação: Função Social (0-100) & 72,5 & 19,3 \\
\hline
\end{tabular}

Tabela 3. Desempenho dos alunos com deficiência nas áreas de autocuidado, mobilidade e função social, segundo a idade. DRE-Penha/São Paulo, 2007- 2009.

\begin{tabular}{|c|c|c|c|c|c|}
\hline & \multicolumn{4}{|c|}{ Idade } & \multirow[b]{2}{*}{ p-valor } \\
\hline & $\begin{array}{l}\text { até } 8 \\
\text { anos }\end{array}$ & $\begin{array}{c}>8 \text { até } 9 \\
\text { anos }\end{array}$ & $\begin{array}{c}>9 \text { até } \\
10 \text { anos }\end{array}$ & $\begin{array}{l}>10 \\
\text { anos }\end{array}$ & \\
\hline Pontuação: Autocuidado (0-100\%) & & & & & 0,351 \\
\hline Média & 85,7 & 83,8 & 88,9 & 90,4 & \\
\hline desvio padrão & 20,4 & 21,3 & 18,2 & 14,6 & \\
\hline Pontuação: Mobilidade (0-100\%) & & & & & $0,261(\mathrm{BF})$ \\
\hline Média & 89,0 & 91,7 & 93,4 & 97,6 & \\
\hline desvio padrão & 24,6 & 24,0 & 20,1 & 11,6 & \\
\hline Pontuação: Função Social (0-100\%) & & & & & 0,529 \\
\hline Média & 76,0 & 69,2 & 72,4 & 72,1 & \\
\hline desvio padrão & 16,9 & 23,8 & 18,4 & 19,0 & \\
\hline
\end{tabular}


A Tabela 5 refere-se à comparação da pontuação obtida segundo os grupos de deficiência (diagnósticos agrupados) entre as diferentes áreas: autocuidado, mobilidade e função social, verificando-se diferenças nas médias obtidas em todas as áreas. Havendo diferença entre as medidas foram realizadas comparações múltiplas (comparação dos diagnósticos dois a dois) para identificar quais categorias apresentaram diferenças entre si. Na área de autocuidado observou-se que houve diferença entre a pontuação média obtida no grupo com deficiência física (menor média) em relação aos demais diagnósticos ( $\mathrm{p}<0,05)$, exceto em relação à DGD e síndromes genéticas; houve, também, diferença entre a pontuação obtida no grupo com DGD em relação ao grupo outras deficiências $(\mathrm{p}=0,048)$.

$\mathrm{Na}$ área de mobilidade observou-se que houve diferença entre a pontuação média obtida no grupo com deficiência física em relação a todos os outros diagnósticos $(\mathrm{p}<0,05)$, verificando-se que para este grupo de crianças (deficiência física) a média de mobilidade foi menor do que os demais grupos. Não foram observadas diferenças entre os demais diagnósticos.

$\mathrm{Na}$ área de função social, houve diferença entre a pontuação média obtida no grupo com outras deficiências (maior média) em relação a todos ou demais diagnósticos $(\mathrm{p}<0,05)$; o grupo com DGD foi o que apresentou a menor média.

\section{Discussão}

Os resultados obtidos, em geral, apresentaram coerência entre desempenho e tipos de deficiências avaliadas, o que foi identificado tanto para cada grupo apontado nos critérios, como individualmente. No autocuidado as crianças com deficiência física e DGD foram as mais comprome-

Tabela 4. Desempenho dos alunos com deficiência nas áreas de autocuidado, mobilidade e função social, segundo a série escolar. DRE-Penha/São Paulo, 2007-2009.

\begin{tabular}{lrrrrr}
\hline & \multicolumn{3}{c}{ Série escolar (criança) } & \\
\cline { 2 - 5 } & $\mathbf{1}^{\mathbf{a}}$ & $\mathbf{2}^{\mathbf{a}}$ & $\mathbf{3}^{\mathbf{a}}$ & $\mathbf{4}^{\mathbf{a}}$ & p-valor \\
\hline $\begin{array}{l}\text { Autocuidado (0-100\%) } \\
\quad \text { Média }\end{array}$ & 77,8 & 84,8 & 89,9 & 95,2 & $<\mathbf{0 , 0 0 1}(\mathbf{B F})$ \\
$\quad$ desvio padrão & 21,7 & 19,9 & 17,8 & 9,5 & \\
Mobilidade (0-100\%) & & & & & $\mathbf{0 , 0 4 9}(\mathbf{B F})$ \\
$\quad$ Média & 90,0 & 89,3 & 93,1 & 99,8 & \\
$\quad$ desvio padrão & 20,5 & 26,1 & 22,2 & 0,8 & $\mathbf{0 , 0 7 6}(\mathbf{B F})$ \\
$\quad$ Função Social (0-100\%) & & & & & \\
$\quad$ Média & 68,0 & 70,0 & 72,2 & 78,3 & \\
$\quad$ desvio padrão & 22,7 & 20,7 & 19,4 & 13,5 & \\
\hline
\end{tabular}

Tabela 5. Desempenho dos alunos nas áreas de autocuidado, mobilidade e função social, segundo grupos de deficiência. DRE-Penha/São Paulo, 2007-2009.

\begin{tabular}{|c|c|c|c|c|c|c|c|c|}
\hline & $\begin{array}{l}\text { Conduta } \\
\text { típica }\end{array}$ & $\begin{array}{l}\text { Deficiência } \\
\text { física }\end{array}$ & DGD & $\begin{array}{l}\text { Múltiplas } \\
\text { deficiências }\end{array}$ & SD & $\begin{array}{c}\text { Síndromes } \\
\text { genéticas }\end{array}$ & $\begin{array}{c}\text { Outras } \\
\text { deficiências }\end{array}$ & p-valor \\
\hline Média & 94,2 & 67,6 & 74,2 & 92,5 & 87,9 & 83,8 & 97,6 & $<0,001(\mathrm{BF})$ \\
\hline desvio padrão & 13,8 & 24,3 & 21,4 & 13,0 & 9,7 & 15,9 & 5,1 & \\
\hline \multicolumn{9}{|c|}{ Escore: Mobilidade $(0-100 \%)$} \\
\hline Média & 100,0 & 62,7 & 97,0 & 98,1 & 99,4 & 96,4 & 99,8 & $<0,001(\mathrm{BF})$ \\
\hline desvio padrão & 0,0 & 36,6 & 8,0 & 10,3 & 1,3 & 7,8 & 0,9 & \\
\hline \multicolumn{9}{|c|}{ Escore: Função Social (0-100\%) } \\
\hline Média & 71,9 & 74,9 & 58,7 & 75,1 & 61,0 & 63,1 & 86,5 & $<0,001(\mathrm{BF})$ \\
\hline desvio padrão & 18,1 & 20,3 & 23,2 & 16,6 & 19,3 & 21,2 & 9,8 & \\
\hline
\end{tabular}


tidas. Aquelas com diagnóstico de conduta típica, deficiência global do desenvolvimento, múltiplas deficiências, Síndrome de Down, Síndromes genéticas e outras apresentaram desempenho menos favorável na função social. Nas crianças com deficiência física, como esperado, o maior problema foi a mobilidade. Chamou a atenção, entretanto, que para todos os grupos a função social foi a mais comprometida, demonstrando que independente do tipo de deficiência há limitações que certamente dificultam o processo de inclusão, mesmo para aquelas condições que não seria esperado, como para deficiência física mesmo que isolada, por exemplo.

No que se refere ao desempenho das crianças em relação à idade e série, segundo o PEDI, pôdese verificar que apenas a série se associou a um aumento de médias obtidas. Estes resultados podem ser explicados pelo fato de que a progressão da criança nas séries é definida e discutida entre a direção da escola e a família e, ainda, que a orientação seja para progressão continuada, alguns alunos se mantinham na mesma série por questões de adaptação, adequação ou opção familiar. Esse procedimento revelou a adoção de uma política mais flexível do regime de progressão continuada adotado pela Secretaria de Educação para as crianças com deficiência. Dessa maneira, algumas crianças foram submetidas a esse regime e foram aprovadas automaticamente e, outras, por opção dos pais e orientação da escola, foram retidas na mesma série escolar, por ter sido avaliado que elas não apresentavam condições de continuidade com um grupo de idade igual, mas de comportamento e instrução diferenciados. Tomando-se como exemplo, uma criança com 10 anos de idade poderia estar numa determinada série porque foi aprovada continuamente ou em outra, com as mesmas habilidades, por ter sido retida. Com maior frequência a opção verificada era de retenção na série, por esse motivo, neste trabalho, verificou-se que o desempenho apresentava maior relação com a série do que com a idade. Com base nas informações obtidas no contato inicial entre a pesquisadora e a direção da escola, conforme descrito na seção métodos, observou-se que a progressão é discutida e definida de forma compartilhada entre pais e equipe da escola, visando a avaliar a melhor opção para cada criança. Também durante o estudo ocorreram casos de pais que pediam para reter a criança com receio de que a mesma apresentasse dificuldade no contato com grupos de crianças maiores ou com a troca de professores. Ainda em relação à distribuição por séries das crianças avaliadas, cabe ressaltar a necessidade de estudos que demonstrem qual a taxa de abandono no grupo de crianças com deficiência, o que possibilitará dimensionar a continuidade e a efetividade do processo inclusivo.

Mantoan ${ }^{14}$ descreve a evolução dos conceitos de incapacidade e inadaptação, em que esta última é resultante da interação entre os hábitos de vida da pessoa e os obstáculos impostos pelo meio, poderá estender-se e alcançar o espaço das escolas e eliminar ou reduzir esses obstáculos do ponto de vista cognitivo. De fato, assim como o meio físico e a arquitetura das escolas não foram planejados para acolher alunos em cadeiras de rodas, o ambiente cognitivo das escolas não está, no geral, preparado para o ensino de pessoas com deficiência mental integradas às normais. O reconhecimento do papel dos fatores ambientais e dos que são internos aos indivíduos, no processo interativo de produção de inadaptações, expressase em todas as inovações conceituais citadas. As características do indivíduo foram durante muito tempo a única meta das intervenções educativas, mas, hoje, nenhum modelo educativo pode ignorar as características do funcionamento das pessoas que apresentam incapacidades intelectuais, sem considerar a interação destas com o meio.

Entende-se por um desenho inclusivo ou universal um conjunto de preocupações, conhecimentos, metodologias e práticas que visam à concepção de espaços, produtos e serviços, utilizáveis com eficácia, segurança e conforto pelo maior número de pessoas possível, independentemente das suas capacidades.

Quanto à utilização do instrumento PEDI, considerou-se que o mesmo contribuiu para a identificação das incapacidades individuais podendo constituir um instrumento útil no acompanhamento do aluno a longo prazo, o que tem sido proposto por outros autores. Os resultados do "Estudo do desempenho funcional de crianças com paralisia cerebral diparética espástica utilizando o PEDI revelaram que o impacto do diagnóstico deste tipo de paralisia cerebral no perfil funcional das crianças é manifestado, principalmente, em atividades que envolvem coordenação bi-manual e nas atividades de transferência. Os autores concluíram que os resultados puderam contribuir para o direcionamento de estratégias de avaliação e de intervenção em crianças portadoras de paralisia cerebral, sendo também uma ferramenta auxiliar para seu processo de inclusão ${ }^{15}$.

Em outro estudo, realizado pelo PROPAI (Programa de promoção e apoio à inclusão de crian- 
ças com deficiência física $)^{11}$ e desenvolvido por professores e acadêmicos do curso de fisioterapia da Universidade Estadual de Londrina, em 2004, foram avaliados 52 alunos em 34 escolas, os quais apresentavam diferentes deficiências. O PEDI foi utilizado com o objetivo de levantar as necessidades para nortear as indicações de adaptações do mobiliário, espaço físico e recursos de baixa tecnologia necessários à mobilidade e participação desses alunos na escola. Concluiu-se que a carência de recursos humanos capacitados e adaptações dos mobiliários e espaço físico são condições frequentes nas escolas, evidenciando a importância da atuação do fisioterapeuta como colaborador no processo educacional de alunos com deficiência física, tanto para favorecer sua acessibilidade/participação, quanto para a capacitação específica dos professores ${ }^{11}$. As questões quanto à acessibilidade podem ser entendidas como a possibilidade de alcance aos espaços físicos, à informação, aos instrumentos de trabalho e estudo, aos produtos e serviços e diz respeito à qualidade de vida de todas as pessoas ${ }^{16}$.

Neste estudo foi observado, também, que no grupo de crianças com deficiência global do desenvolvimento, houve grande comprometimento do autocuidado e da função social, esta última já esperada por ser característica do transtorno. Vários estudos sobre o desfecho no longo prazo de crianças e jovens com autismo sugerem que aproximadamente dois terços das crianças autistas apresentam um desfecho pobre (incapazes de viver independentemente) e que somente um terço é capaz de atingir algum grau de independência pessoal e de autossuficiência como adultos; entre estes, a maioria pode ter um desfecho razoável (ganhos sociais, educacionais ou vocacionais a despeito de dificuldades comportamentais e de outra ordem), ao passo que uma minoria (cerca de um décimo de todos os indivíduos com autismo) pode ter um bom desfecho (ter capacidade de exercer atividade profissional com eficiência e ter vida independente) ${ }^{17}$. Os estudos acerca do desfecho dessas crianças são importantes por apontarem suas condições de vida, mas não devem servir como profecias que desencorajem sua inclusão na sociedade.
A identificação de comprometimento da função social em todos os grupos de crianças chamou a atenção para as estratégias a serem utilizadas nas escolas de forma compartilhada com suas famílias. Simultaneamente a uma atuação específica voltada para a deficiência da criança, e que o PEDI pode apontar detalhadamente, esforços nos sentido de favorecer a socialização da criança e contribuir para o processo de inclusão constituem grandes desafios e puderam ser revelados de forma mais objetiva por meio da aplicação deste instrumento.

Luna et al. ${ }^{18}$ realizaram um estudo com 278 escolares com múltiplas deficiências. Aproximadamente uma em cada cinco mães considerava seus filhos com problemas de relacionamento familiar, escolar ou social. Nesse estudo, os autores verificaram que quase metade dessas crianças $(44,6 \%)$ estava excluída de qualquer tipo de escola e que o baixo nível sociocultural predominante na população estudada e a desinformação quanto aos direitos de seus filhos dificultava aos pais perceberem a exclusão deles. As escolas regulares influenciaram positivamente na socialização das crianças com deficiências múltiplas.

É reconhecido que a educação da criança com deficiência é uma atividade complexa, pois exige adaptações de ordem curricular que requerem cuidadoso acompanhamento dos educadores e pais. Frequentar a escola permite à criança com deficiência adquirir, progressivamente, conhecimentos cada vez mais complexos, os quais serão exigidos pela sociedade e cujas bases são indispensáveis para a formação do indivíduo ${ }^{19}$.

\section{Conclusão}

A função social esteve comprometida em todos os grupos de deficiência, sendo mais acentuada nas crianças com deficiência global do desenvolvimento, síndrome de Down e síndromes genéticas, demonstrando que a presença da deficiência compromete de forma significativa o processo de inclusão. A utilização de instrumentos que possam identificar limitações individuais visando ao direcionamento de intervenções da parte da escola e da família podem ser úteis no acompanhamento dessas crianças. 


\section{Colaboradores}

FM Teles, R Resegue e RF Puccini participaram igualmente de todas as etapas de elaboração do artigo.

\section{Referências}

1. Carneiro MA. O acesso de alunos com deficiência às escolas e classes comuns. Possibilidades e limitações. Petrópolis: Vozes; 2007.

2. Brasil. Política Nacional de Saúde da Pessoa Portadora de deficiência. [página na Internet]. [acessado 2010 jan 18]. Disponível em: http://portal.saude. gov.br/portal/arquivos/pdf/manual2.pdf

3. Brasil. Ministério da Justiça. Declaração de Salamanca e linhas de ação sobre necessidades educativas especiais. Brasília: Corde; 1994.

4. Mazzota MJS. Educação Especial no Brasil: História e Políticas Públicas. 4a Edição. São Paulo: Cortez; 2003.

5. Instituto Brasileiro de Geografia e Estatística (IBGE). Censo Demográfico 2000. [página na Internet]. [acessado 2010 jan 16]. Disponível em: http://www. ibge.gov.br/home/presidencia/noticias/27062003 censo.shtm

6. Angelucci CB. Uma inclusão nada especial. Aprimorações da política de inclusão de pessoas com necessidades especiais na rede pública de educação fundamental do Estado de São Paulo. São Paulo: Universidade de São Paulo; 2002.

7. Araújo VC. Infância e educação inclusiva. Florianopolis: Perspectiva; 2005.

8. Mont D, Loeb MD. Developing indicators to assess the impact of public healt. Interventions on the lives of peoples with disabilities. HDNSP - The World Bank, 2008. p. 8-14.

9. Lima PA. Educação Inclusiva e Igualdade Social. São Paulo: Avercamp; 2006

10. Carneiro MA. O acesso de alunos com deficiência às escolas e classes comuns. Possibilidades e limitações. Petrópolis: Vozes; 2007.

11. Alpino MAS. Propai - Programa de promoção e apoio a inclusão de crianças com deficiência física: Um projeto de extensão universitária. Rev. Estação; 2006.
12. Mancini MC. Inventário de avaliação pediátrica de incapacidade (PEDI): manual da versão brasileira adaptada. Belo Horizonte: UFMG; 2005.

13. McDowell I, Newell C. Measuring Health. $2^{\text {nd }}$ Edition. New York: Oxford University Press; 1996.

14. Mantoan MTE. Educação escolar de deficientes mentais: Problemas para a pesquisa e o desenvolvimento. Campinas. Caderno Cedes 1998; 19(46):3-4.

15. Schwartzman JS. Estudo do desempenho funcional de crianças com paralisia cerebral diparética espástica utilizando o Pediatric Evaluation of Disability Inventory (PEDI). São Paulo: Arquivos brasileiros de paralisia cerebral; 2004.

16. Pupo DT, Melo AM, Ferrés SP. Acessibilidade. Discurso e prática no cotidiano da biblioteca. Lab. de acessibilidade. Campinas: Unicamp: Biblioteca Central Cesar Lattes; 2006.

17. Klin A. Autismo e síndrome de Asperger: uma visão geral. Rev. Brasileira de Psiquiatria 2006; 28(Supl. 1):S3-11.

18. Luna G, Leal D, Carneiro A, Rached S, Siqueira J, Ventura L. Inclusóes social, familiar e escolar de pacientes com múltiplas deficiências atendidos em centros de referências de Pernambuco. Pernambuco: Univ. Fed. Pernambuco; 2005.

19. Schwartzman JS, organizador. Sindrome de Down. São Paulo: Mackenzie, Memnon; 1999.

Artigo apresentado em 11/02/2012

Aprovado em 05/03/2012

Versão final apresentada em 21/03/2012 